\title{
Genetic Diversity of Landraces and Improved Varieties of Rice (Oryza sativa L.) in Taiwan
}

\author{
Ai-ling Hour ${ }^{1}$, Wei-hsun Hsieh², Su-huang Chang ${ }^{2}$, Yong-pei Wu ${ }^{3}$, Han-shiuan Chin ${ }^{2}$ and Yann-rong Lin ${ }^{2 *}$ (D)
}

\begin{abstract}
Background: Rice, the most important crop in Asia, has been cultivated in Taiwan for more than 5000 years. The landraces preserved by indigenous peoples and brought by immigrants from China hundreds of years ago exhibit large variation in morphology, implying that they comprise rich genetic resources. Breeding goals according to the preferences of farmers, consumers and government policies also alter gene pools and genetic diversity of improved varieties. To unveil how genetic diversity is affected by natural, farmers', and breeders' selections is crucial for germplasm conservation and crop improvement.

Results: A diversity panel of 148 rice accessions, including 47 cultivars and 59 landraces from Taiwan and 42 accessions from other countries, were genotyped by using 75 molecular markers that revealed an average of 12.7 alleles per locus with mean polymorphism information content of 0.72 . These accessions could be grouped into five subpopulations corresponding to wild rice, japonica landraces, indica landraces, indica cultivars, and japonica cultivars. The genetic diversity within subpopulations was: wild rices > landraces > cultivars; and indica rice > japonica rice. Despite having less variation among cultivars, japonica landraces had greater genetic variation than indica landraces because the majority of Taiwanese japonica landraces preserved by indigenous peoples were classified as tropical japonica. Two major clusters of indica landraces were formed by phylogenetic analysis, in accordance with immigration from two origins. Genetic erosion had occurred in later japonica varieties due to a narrow selection of germplasm being incorporated into breeding programs for premium grain quality. Genetic differentiation between early and late cultivars was significant in japonica $\left(F_{S T}=0.3751\right)$ but not in indica $\left(F_{S T}=\right.$ 0.0045), indicating effects of different breeding goals on modern germplasm. Indigenous landraces with unique intermediate and admixed genetic backgrounds were untapped, representing valuable resources for rice breeding.

Conclusions: The genetic diversity of improved rice varieties has been substantially shaped by breeding goals, leading to differentiation between indica and japonica cultivars. Taiwanese landraces with different origins possess various and unique genetic backgrounds. Taiwanese rice germplasm provides diverse genetic variation for association mapping to unveil useful genes and is a precious genetic reservoir for rice improvement.
\end{abstract}

Keywords: Genetic diversity, Landraces, Rice, Taiwan

\footnotetext{
* Correspondence: ylin@ntu.edu.tw

${ }^{2}$ Department of Agronomy, National Taiwan University, Taipei 10617, Taiwan

Full list of author information is available at the end of the article
}

\section{Springer Open}

(c) The Author(s). 2020 Open Access This article is licensed under a Creative Commons Attribution 4.0 International License, which permits use, sharing, adaptation, distribution and reproduction in any medium or format, as long as you give appropriate credit to the original author(s) and the source, provide a link to the Creative Commons licence, and indicate if changes were made. The images or other third party material in this article are included in the article's Creative Commons licence, unless indicated otherwise in a credit line to the material. If material is not included in the article's Creative Commons licence and your intended use is not permitted by statutory regulation or exceeds the permitted use, you will need to obtain permission directly from the copyright holder. To view a copy of this licence, visit http://creativecommons.org/licenses/by/4.0/. 


\section{Background}

Asian cultivated rice (Oryza sativa L.), feeding more than 90\% of the human population in Asia, is one of the world's most important crops. Wild ancestors and landraces with rich genetic diversity and wide adaptation to various environments provide valuable and useful genetic resources for crop improvement (Dwivedi et al. 2016; Kovach and McCouch 2008; Sang and Ge 2013). Natural germplasm preserved by in situ and/or ex situ conservation is in urgent need of systematic evaluation to unveil new genes or alleles to incorporate into breeding programs for crop improvement. For rice, the most well-known germplasm conservation center is the International Rice Genebank Collection at the International Rice Research Institute (IRRI). Single nucleotide polymorphisms (SNPs) and structural variants revealed by resequencing accelerate research on genetic diversity, evolution, association studies of genotypes and phenotypes and allele mining (Huang et al. 2010; Li et al. 2014; Wang et al. 2018; Zhao et al. 2018).

Oryza sativa was domesticated from wild rice, $O$. nivara or O. rufipogon (Chang 1976; Khush 1997), and two distinct varietal groups, ssp. indica and ssp. japonica, are well recognized and dated as Hsien (秈) and Keng (稉), respectively, in the Hang dynasty about 2000 years ago (Chou 1948; Wang et al. 2018). Distinct morphology and post-reproductive barriers between ssp. indica and ssp. japonica were first thought to have resulted from independent domestication by different ancient populations or a single domestication with multiple origins recently (Londo et al. 2006; Choi et al. 2017; Choi and Purugganan 2018). The genetic diversity of $O$. sativa was dramatically reduced by bottleneck effects of selective sweeps in early domestication (Caicedo et al. 2007; Kovach and McCouch 2008). Landraces which are morphologically recognizable and have historical origins exhibit lower genetic diversity than wild relatives but higher than modern cultivars because of adaptation to local environments and diversity of farmers' preferences (Pusadee et al. 2009; Thomson et al. 2007). The allele richness of landraces was, in general, about 30\% higher than that of cultivars (Kovach and McCouch 2008; Zhang et al. 2009) and landraces possess a wealth of abiotic tolerances, biotic resistances and other superior characters. Taken together, investigation of morphological, physiological, and genetic diversity of landraces will provide valuable information and resources for modern rice breeding.

In Taiwan, archaeological evidence shows that rice has been cultivated by indigenous people for more than 5000 years (Hu 1993). Excavated grains exhibit various sizes and shapes and resemble tropical japonica and indica rice (Hsieh et al. 2011). In the early seventeenth century, immigrants from two provinces of southeast China, Fujian and Guangdong, brought indica landraces to Taiwan. In the late nineteenth century, approximately 1197 collections of temperate japonica rice were introduced from Japan (Iso 1964). Sixty Taiwanese landraces have been widely used in rice research and breeding, revealed by 16 domestication-syndrome genes (Hsieh et al. 2011). Taiwanese landraces have contributed significantly to modern indica and temperate japonica rice breeding in Asian countries. The most renowned example is IR8, the miracle rice with high yield that mitigated a food crisis in the 1970s and evoked a Green Revolution in Asia, which inherited the semi-dwarf allele $(s d 1)$ from Taiwanese landrace Dee-GeoWoo-Gen (DGWG) (Evenson and Gollin 2003). Indeed, the DGWG allele has been extensively applied to improve grain yield of both indica and japonica varieties in the past 50 years (Sasaki et al. 2002; Asano et al. 2011; Zhao et al. 2010). Taichung 65 (TC65), an old japonica cultivar, inherited null alleles of two photoperiodic genes, Ehd1 and Hd1, from landraces and has been extensively applied in modern rice breeding and in studying flowering in response to day length (Doi et al. 2004; Hsieh et al. 2011; Lin 1991; Wei et al. 2016; Yano et al. 2000).

Many modern cultivars integrate temperate japonica and indica rice toward meeting the major demands of daily dining and traditional food processing in Taiwan. A lot of genetic variation is found in Taiwanese rice germplasm because of natural selection for adaptation to various environments, noting that Taiwan encompasses tropical and subtropical zones in a broad range of altitudes $(0-3952 \mathrm{~m})$. The genetic diversity of Taiwanese rice germplasm, originating from different geographic areas and admixed by humans in different epochs, is expected to be high (Chin et al. 2016).

To unravel admixing of rice germplasm imposed by natural and artificial selection is important for basic scientific research and breeding, each relying on information about genetic diversity and population structure. In this study, a diversity panel of 148 accessions, including 53 modern varieties, 83 landraces, and 12 wild rice originating from Taiwan, Japan, China and countries of southeast Asia and south Asia, was genotyped with 75 markers to assess genetic diversity and population structure, conducting principal coordinate analysis (PCoA) and producing a phylogenetic tree. In addition, Taiwanese landraces are scrutinized, gaining insight into their significant roles in genetic and breeding research.

\section{Results}

\section{Genetic Diversity of Polymorphic Markers}

A total of 953 alleles were detected from 75 DNA markers, including 49 simple sequence repeat (SSR), 6 sequence-tagged site (STS) and 20 indel markers, across the diversity panel of 148 rice accessions, including 12 wild rices, 83 landraces, 24 indica cultivars, and 29 japonica cultivars (Additional file 1: Table S1). The allele 
number per locus ranged from 3 to 37 with an average of 12.7 , and the majority of markers revealed 6-15 alleles. (Additional file 2: Figure S1a, Additional file 1: Table S2). Eight markers, RM472, RM2334, RM4108, CH0509, P17G10-24, RM1761, RM4154 and RM5708, were highly polymorphic with more than 20 alleles detected (Additional file 1: Table S2). Polymorphism information content (PIC) values ranged from 0.18 to 0.95 with an average of 0.72 , and $66(88 \%), 8(10.7 \%)$ and $1(1.3 \%)$ markers were highly, moderately and slightly informative, with $\mathrm{PIC} \geq 0.5,0.5>\mathrm{PIC} \geq 0.25$, and $<0.25$, respectively (Additional file 2: Figure S1b, Additional file 1: Table S2). Overall, these 75 markers provided plentiful allele information to assess genetic diversity, population structure, and genetic distances of this rice diversity panel.

\section{Genetic Structure and Diversity of Subpopulations}

These 148 accessions could be divided into two subpopulations according to inferred population structures, with $\Delta K$ values found to be highest at $K=2$ by STRUCT URE analysis (Additional file 2: Figure S2). The japonica group constituted 62 accessions, including one wild rice, O. rufipogon, 29 Taiwanese japonica cultivars and 32 landraces from Taiwan, Japan, and China. All 29 Taiwanese japonica cultivars, except Kaohsiung 145, shared 99.9\% ancestry, indicating a consistent genetic background. The indica group contained 86 accessions, including 24 cultivars, 51 landraces, and 11 wild rices.
Admixture, simulated single genetic background less than $80 \%$, was frequently observed in wild rice, except nivara-2 and rufipogon-21, but occasionally in cultivars (Taichung Sen 17 and Basmati 385) and landraces (Tangengenrankatsu and Chin-Men-Tou-Men-Hung-Mi) (Additional file 2: Figure S3a).

To further subdivide this germplasm, five subpopulations, denoted PopI to PopV, were obtained as $K=5$ was the optimum number of subpopulations identified by STRUCTURE analysis (Table 1, Additional file 2: Figure $\mathrm{S} 3 \mathrm{~b})$. The japonica group was subdivided into two subpopulations, PopII and PopIII. Most japonica landraces were grouped into PopII, including 23 and 2 accessions originating from Taiwan and China, respectively. PopIII contained 36 japonica accessions, including 29 cultivars sharing 89-99\% uniform subpopulation background; and 6 landraces of which (Burieuraozu, Paotsupagaiahon, Tangengenrankatsu, Nabohai, Unknown 1 and Sinceyauo), were admixtures with $44-83 \%$ of subpopulation PopIII genetic background. The Taiwanese upland $j a-$ ponica landrace, Tangengenrankatsu, containing some indica genetic background, was particularly interesting.

The indica group was subdivided into three subpopulations, PopI, PopIV and PopV (Table 1, Additional file 2: Figure S3b). Twenty accessions, including 17 Taiwanese cultivars, Basmati 370, Kasalasth, and IR64, were grouped in PopI. Four accessions (Tainung Sen 22, Kaohsiung Sen 7, Taichung Sen Glutinous 2, and Basmati

Table 1 The accessions of five subpopulations grouped by STRUCTURE analysis

\begin{tabular}{|c|c|c|}
\hline Subpopulation & Number & Accession $^{a}$ \\
\hline Popl & 20 & $\begin{array}{l}\text { Tai Sen } 2(\mathrm{IC}) \text {, Tainung Sen } 19(\mathrm{IC}) \text {, Taichung Sen Glutinous } 2(\mathrm{IC}) \text {, Taichung Sen } 10 \text { (IC), Tai Sen } 1(\mathrm{IC}) \text {, Tainung Sen } 20 \text { (IC), } \\
\text { Taichung Sen } 17(\mathrm{IC}) \text {, Tainung Sen } 12(\mathrm{IC}) \text {, IR } 64 \text { (IC), Taichung Native } 1 \text { (IC), Tainung Sen } 14(\mathrm{IC}) \text {, Chianung Sen } 6 \text { (IC), } \\
\text { Taichung Sen Glutinous } 1(\mathrm{IC}) \text {, Kasakasth (IC), Tainung Sen } 21 \text { (IC), Tainung Sen } 18 \text { (IC), Kaohsiung Sen } 7 \text { (IC), Basmati } 370 \\
\text { (IC), CNY922401 (IC), Tainung Sen 22(IC). }\end{array}$ \\
\hline Popll & 25 & 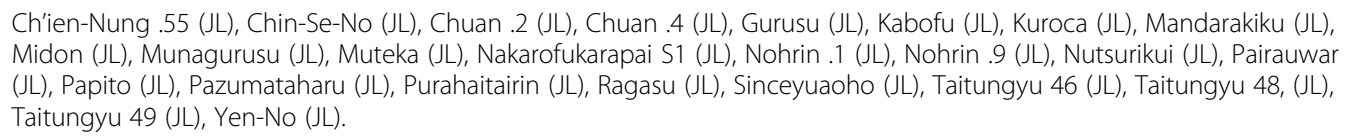 \\
\hline PopllI & 36 & 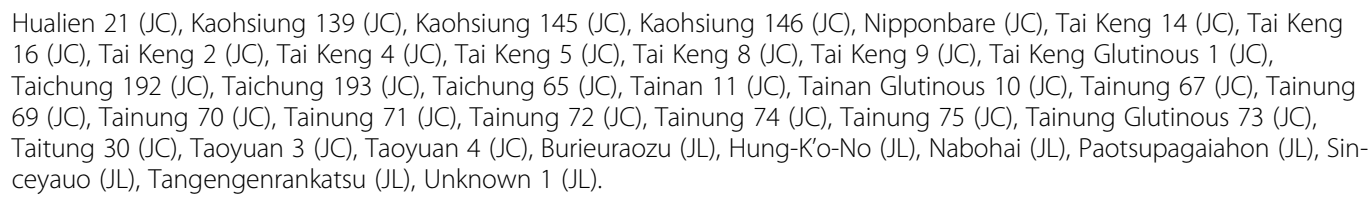 \\
\hline PoplV & 53 & $\begin{array}{l}\text { Dular (IC), Tien-Lai (IL), T' ai-Tung-Shung-Tung-Ch'ung (IL), T' ai-Tung-Ta-Ma-Li-Wu-Chan (IL), Unknown } 3 \text { (IL), Shang-Chi- } \\
\text { Tsao-Tao (IL), Chi-Shih-Jih (IL), San-Pei (IL), Baridon (IL), Wu-K'o-Tsao-Tzu (IL), Lin-Mang (IL), I-Kung-Pao (IL), Pai-Chan (IL), } \\
\text { Chu-Tzu (IL), Parahainakoru (IL), Lei-Ch'ui (IL), Ching-Yu (IL), Kao-Chueh-Wu-Chan (IL), Pai-K' o-Tsao-Tzu (IL), Pai-K'o-P' u- } \\
\text { Chan (IL), Shiau-No (IL), Jao-Yao (IL), Ch'ih-K'O (IL), Chung-Chiu No (IL), Ko-Tzu (IL), Tsui-Lo-Ku (IL), Wu-K'o (IL), Hua-Lou (IL), } \\
\text { O-Nung .3 (IL), Hopots Utaiyaru (IL), Tuan-Li-No (IL), Cheng-wu-Chan (IL), Tan-Yang-No (IL), Unknown (IL), Cheng-Ching-Yu } \\
\text { (IL), Chang-Hsu-Ku (IL), Wu-Chan (IL), Fukutomi (IL), Tsao-Chiu-Ku (IL), Yin-Yu-Tze (IL), Hsiao-Ko-Tzu (IL), Shuang-Chiang- } \\
\text { Tsao-1 (IL), Wu-No-Tao (IL), Hung-No (IL), Yuan-Li (IL), Kaisentetsuchitsu (IL), Napatsupai S3 (IL), Pai-K'O-Yuan-Li (IL), Lu- } \\
\text { Tao3036 (IL), Pakaikauneku (IL), Chin-Men-Tou-Men-Hung-Mi (IL), Pai-Mi-Fen (IL), O. rufipogon-21 (W). }\end{array}$ \\
\hline PopV & 14 & $\begin{array}{l}\text { Basmati } 385 \text { (IC), Taichung Sen } 2 \text { (IC), Taichung Sen } 3(\mathrm{IC}) \text {, O. nivara-2 (W), O. nivara-5 (W), O. nivara-6 (W), O. nivara-7 (W), } \\
\text { O. rufipogon-10 (W), O. rufipogon-12 (W), O. rufipogon-15 (W), O. rufipogon-16 (W), O. rufipogon-18 (W), O. rufipogon-19 } \\
\text { (W), O. rufipogon-20 (W). }\end{array}$ \\
\hline
\end{tabular}

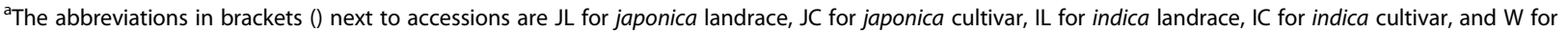
wild rice 
370) admixed with PopIV were noted. Most of the 53 accessions of PopIV, except Dular and O. rufipogon-21, were indica landraces. Two Taiwanese landraces (Pakaikauneku and Pai-Mi-Fen) and a Chinese landrace (Chin-Men-TouMen-Hung-Mi) were admixed with PopII. PopV contained 11 wild rice accessions and 3 indica cultivars (Taichung Sen 2, Taichung Sen 3, and Basmati 385).

The genetic diversity of each subpopulation was evaluated by using 4 parameters, mean allele number per locus, major allele frequency per locus, gene diversity and PIC value (Table 2). PopV, consisting of 11 wild rice and 3 indica cultivars, displayed the most diverse genetic background, revealed by the highest mean allele number (6.91), Nei's gene diversity (0.74) and mean PIC value (0.71) but the lowest major allele frequency per locus (0.37) (Table 2). On the other hand, PopIII, consisting of 29 japonica cultivars and 7 japonica landraces, exhibited the lowest genetic diversity. The genetic diversities of these five subpopulations were PopV, wild rices > PopII, japonica landraces > PopIV, indica landraces > PopI, indica cultivars > PopIV, japonica cultivars. While it was anticipated that landraces would be generally more diverse than cultivars, it was noteworthy that the genetic diversity of indica cultivars was higher than that of japonica cultivars, while the genetic diversity of indica landraces was slightly lower than that of japonica landraces albeit more indica accessions were assessed (Table 2).

\section{Genetic Divergence in Asian Cultivated O. sativa}

The genetic diversity of the 136 O. sativa accessions evaluated was relatively high as revealed by mean allele number (11.01), mean gene diversity (0.74) and mean PIC (0.70) (Table 3). The highly diverse 83 landraces contributed the majority of genetic variation in this panel. Genetic diversity, in general, was higher in the indica than the japonica group; however, japonica landraces exhibited higher variation than indica landraces. The genetic diversity of cultivars was relatively narrow as compared to landraces, and japonica cultivars had the least variation (Table 3).
Relatively high $F_{\mathrm{ST}}$ values of 0.3084 and 0.3040 was observed between indica and japonica in all $O$. sativa accessions and in landraces, respectively (Table 3). In modern breeding under intensified directional selection, indica and japonica cultivars are even more diversified from each other as revealed by the highest $F_{\mathrm{ST}}$ value (0.4200). On the other hand, there was less divergence between cultivars and landraces both in indica and japonica.

\section{Divergence of Taiwanese Rice Germplasm}

In the collection of 106 Taiwanese accessions, the genetic diversity of indica accessions was higher than that of japonica ones, and the genetic diversity of landraces was also higher than cultivars (Table 4). The genetic diversity of indica landraces was not obviously different from japonica landraces; however, indica cultivars exhibited greater diversification than japonica cultivars. Taiwanese cultivars were divided into 'early' cultivars or 'late' cultivars consistent with the government policy of rice breeding goals changing from yield (early) to premium grain quality (late) in 1981. The 25 late japonica cultivars exhibited larger genetic diversity than 3 early japonica cultivars; nevertheless, the difference was not statistically significant difference between early and late indica cultivars (Table 4).

For the Taiwanese accessions, great differentiation between indica and japonica types was indicated by high $F_{\mathrm{ST}}(0.3181)$, with Taiwanese landraces similar to this overall trend $\left(F_{\mathrm{ST}}=0.3142\right)$ but higher differentiation between indica and japonica cultivars $\left(F_{\mathrm{ST}}=0.4251\right)$ (Table 4). Less differentiation between Taiwanese japonica cultivars and landraces $\left(F_{\mathrm{ST}}=0.1995\right)$ and indica cultivars and landraces $\left(F_{\mathrm{ST}}=0.1344\right)$ were observed. The late indica cultivars were not differentiated from the early indica cultivars $\left(F_{\mathrm{ST}}=0.0045\right)$; however, the late $j a$ ponica cultivars were significantly differentiated from the early japonica cultivars $\left(F_{\mathrm{ST}}=0.3751\right)$.

\section{Relatedness Based on Genetic Distances}

The 148 accessions could be separated into two groups corresponding to indica and japonica by 2-dimensional

Table 2 Genetic diversity parameters of five subpopulations

\begin{tabular}{llllll}
\hline Population $^{\mathbf{a}}$ & Sample size & Mean allele number /locus & Major allele frequency/locus & Nei's gene diversity & Mean PIC $^{\mathbf{b}}$ value $^{-}$ \\
\hline All subpopulations & 148 & 12.71 & 0.36 & 0.75 & 0.72 \\
Popl (IC) & 20 & 4.16 & 0.62 & 0.49 & 0.45 \\
Popll (JL) & 25 & 5.89 & 0.50 & 0.60 & 0.57 \\
PopIII (JC) & 36 & 4.64 & 0.65 & 0.47 & 0.43 \\
PopIV (IL) & 53 & 6.68 & 0.53 & 0.58 & 0.54 \\
PopV (W) & 14 & 6.91 & 0.37 & 0.74 & 0.71
\end{tabular}

${ }^{a}$ The majority of accessions in Popl, Popll, Poplll, PopIV and PopV are indica cultivar, japonica landrace, japonica cultivar, indica landrace, and wild rice, respectively ${ }^{\mathrm{b}} \mathrm{PIC}$ is the abbreviation for polymorphism information content 
Table 3 Genetic diversity and divergence in O. sativa

\begin{tabular}{|c|c|c|c|c|c|c|}
\hline Group & Number & Mean allele number/locus & Major allele frequency/locus & Mean gene diversity & Mean PIC a value & $F_{\mathrm{ST}}^{\mathrm{b}}$ \\
\hline All O. sativa & 136 & 11.01 & 0.36 & 0.74 & 0.70 & 0.3084 \\
\hline Indica & 75 & 8.31 & 0.50 & 0.61 & 0.58 & \\
\hline Japonica & 61 & 7.05 & 0.54 & 0.59 & 0.55 & \\
\hline All landraces & 83 & 9.57 & 0.39 & 0.72 & 0.69 & 0.3040 \\
\hline Indica & 51 & 6.52 & 0.53 & 0.58 & 0.54 & \\
\hline Japonica & 32 & 6.25 & 0.50 & 0.60 & 0.57 & \\
\hline All cultivars & 53 & 7.28 & 0.44 & 0.68 & 0.64 & 0.4200 \\
\hline Indica & 24 & 5.43 & 0.57 & 0.56 & 0.52 & \\
\hline Japonica & 29 & 3.65 & 0.68 & 0.43 & 0.39 & \\
\hline All indica & 75 & 8.31 & 0.50 & 0.61 & 0.58 & 0.1166 \\
\hline Cultivars & 24 & 5.43 & 0.57 & 0.56 & 0.52 & \\
\hline Landraces & 51 & 6.52 & 0.53 & 0.58 & 0.54 & \\
\hline All japonica & 61 & 7.05 & 0.54 & 0.59 & 0.55 & 0.1913 \\
\hline Cultivars & 29 & 3.65 & 0.68 & 0.43 & 0.39 & \\
\hline Landraces & 32 & 6.25 & 0.50 & 0.60 & 0.57 & \\
\hline
\end{tabular}

${ }^{\mathrm{a}} \mathrm{PIC}$ is the abbreviation for polymorphism information content

${ }^{\mathrm{b}}$ Fixation index $\left(F_{\mathrm{ST}}\right)$ indicates genetic differentiation between two subpopulations by the reduction of heterozygosity due to genetic drift and / or selection

Table 4 Genetic diversity and divergence in Taiwanese germplasm

\begin{tabular}{|c|c|c|c|c|c|c|}
\hline Group & Number & $\begin{array}{l}\text { Mean allele number/ } \\
\text { locus }\end{array}$ & $\begin{array}{l}\text { Major allele frequency/ } \\
\text { locus }\end{array}$ & $\begin{array}{l}\text { Mean gene } \\
\text { diversity }\end{array}$ & $\begin{array}{l}\text { Mean PIC } \\
\text { value }\end{array}$ & $F_{\mathrm{ST}}$ \\
\hline All Taiwanese accessions & 106 & 10.19 & 0.36 & 0.74 & 0.70 & 0.3181 \\
\hline Indica & 55 & 7.41 & 0.50 & 0.61 & 0.57 & \\
\hline Japonica & 51 & 6.43 & 0.56 & 0.57 & 0.54 & \\
\hline All Taiwanese landraces & 59 & 8.55 & 0.39 & 0.72 & 0.68 & 0.3142 \\
\hline Indica & 36 & 5.73 & 0.53 & 0.58 & 0.53 & \\
\hline Japonica & 23 & 5.37 & 0.52 & 0.58 & 0.55 & \\
\hline All Taiwanese cultivars & 47 & 6.84 & 0.46 & 0.66 & 0.62 & 0.4251 \\
\hline Indica & 19 & 4.81 & 0.58 & 0.54 & 0.51 & \\
\hline Japonica & 28 & 3.63 & 0.68 & 0.42 & 0.38 & \\
\hline Taiwanese indica accessions & 55 & 7.41 & 0.50 & 0.61 & 0.57 & 0.1344 \\
\hline Cultivars & 19 & 4.81 & 0.58 & 0.54 & 0.51 & \\
\hline Landraces & 36 & 5.73 & 0.53 & 0.58 & 0.53 & \\
\hline $\begin{array}{l}\text { Taiwanese japonica } \\
\text { accessions }\end{array}$ & 51 & 6.43 & 0.56 & 0.57 & 0.54 & 0.1995 \\
\hline Cultivars & 28 & 3.63 & 0.68 & 0.42 & 0.38 & \\
\hline Landraces & 23 & 5.37 & 0.52 & 0.58 & 0.55 & \\
\hline All Taiwanese indica cultivars & 19 & 4.81 & 0.58 & 0.54 & 0.51 & 0.0045 \\
\hline Early cultivars ${ }^{a}$ & 7 & 3.29 & 0.59 & 0.52 & 0.47 & \\
\hline Late cultivars & 12 & 3.93 & 0.61 & 0.51 & 0.47 & \\
\hline $\begin{array}{l}\text { All Taiwanese japonica } \\
\text { cultivars }\end{array}$ & 28 & 3.63 & 0.68 & 0.42 & 0.38 & 0.3751 \\
\hline Early cultivars & 3 & 1.72 & 0.76 & 0.29 & 0.24 & \\
\hline Late cultivars & 25 & 3.47 & 0.68 & 0.42 & 0.38 & \\
\hline
\end{tabular}

${ }^{a}$ The early and late cultivars were released before and after 1981, respectively 
PCoA analysis, in which the first and the second dimension explained $18.1 \%$ and $7.7 \%$ of variation, respectively (Fig. 1). Japonica accessions were distinct from indica accessions, and japonica accessions were distributed more sparsely than indica accessions. The indica cultivars could be distinguished from indica landraces by the third dimension, accounting for $3.12 \%$ of variation (Additional file 2: Figure S4). The cultivars were more closely aggregated than landraces for indica and japonica, indicating more similar genetic background. Three landraces (Tangengenrankatsu, Pakaikauneku and Hsiao-K'o-Tzu) and 2 cultivars (Taichung Sen 2 and Taichung Sen 3) were close to wild rice.

The unrooted phylogenetic tree according to Nei's genetic distances revealed two distinct major clusters, indica and japonica, indicated by red and blue branches, respectively (Fig. 2). The indica cluster could be further subdivided into 7 clades, Clade I to VII, and the japonica cluster could be subdivided into 5 clades, Clade VIII to XII. The indica cultivars were grouped in Clade V and distinguished from the indica landraces, which formed one group with 4 distinct clades, Clade I - IV. Clades VIII, IX, X and XII were primarily comprised of japonica landraces. On the other hand, japonica cultivars entirely constituted Clade XI, with the exception that Kaohsiung 145 was closely-related to three japonica landraces, Burieuraozu, Mandarakiku and Papito. These results show that the genetic background of modern indica and japonica cultivars have deviated from those of traditional landraces under intensive selection for breeding goals of high yield and premium grain quality. Finally, wild rice, indicated with a black branch, could not form a distinct group and fell into intermediate locations in the phylogenetic tree. One japonica landrace, Tangengenrankatsu, and four indica cultivars (Basmati
385, Taichung Sen 2, Taichung Sen 3, and CNY922401) also fell into intermediate locations allied with wild rice.

\section{Discussion}

Genetic Diversity Revealed by Assessment of Molecular

\section{Markers}

Genetic diversity evaluated by molecular markers provides useful and fundamental information for crop improvement. Among an assortment of markers, SSRs exhibiting relatively high polymorphism level per locus provide rich allelic information for genetic diversity analysis. A currently-preferred phylogenetic relationship of rice germplasm was established by using SSRs that discerned 5 major groups - specifically, aus, indica, aromatic, temperate japonica and tropical japonica (Garris et al. 2005). Phylogenetic trees of rice germplasm using genome-wide SNPs or structural variants were in accordance with these 5 groups (Wang et al. 2018; McNally et al. 2009; Fuentes et al. 2019). In the present study, a total of 953 alleles were detected by 75 polymorphic markers, varying from 3 to 37 alleles per locus with an average of 12.7 (Additional file 2: Figure S1, Additional file 1: Table S2), which was higher than other studies (Chakhonkaen et al. 2012; Jin et al. 2010; Nachimuthu et al. 2015). PIC values, which are good indicators of marker polymorphism levels, were in the range of 0.18 to 0.95 , with a mean of 0.72 , higher than those reported in European (0.49), Chinese (0.42) and Indonesian (0.66) rice germplasm, respectively (Courtois et al. 2013; Jin et al. 2010; Thomson et al. 2007). Moreover, 66 of 75 markers $(88 \%)$ were considered highly informative with PIC values $>0.5$ (Additional file 2: Figure S1). Thus, these 75 markers provided rich allelic information for genetic diversity analysis.

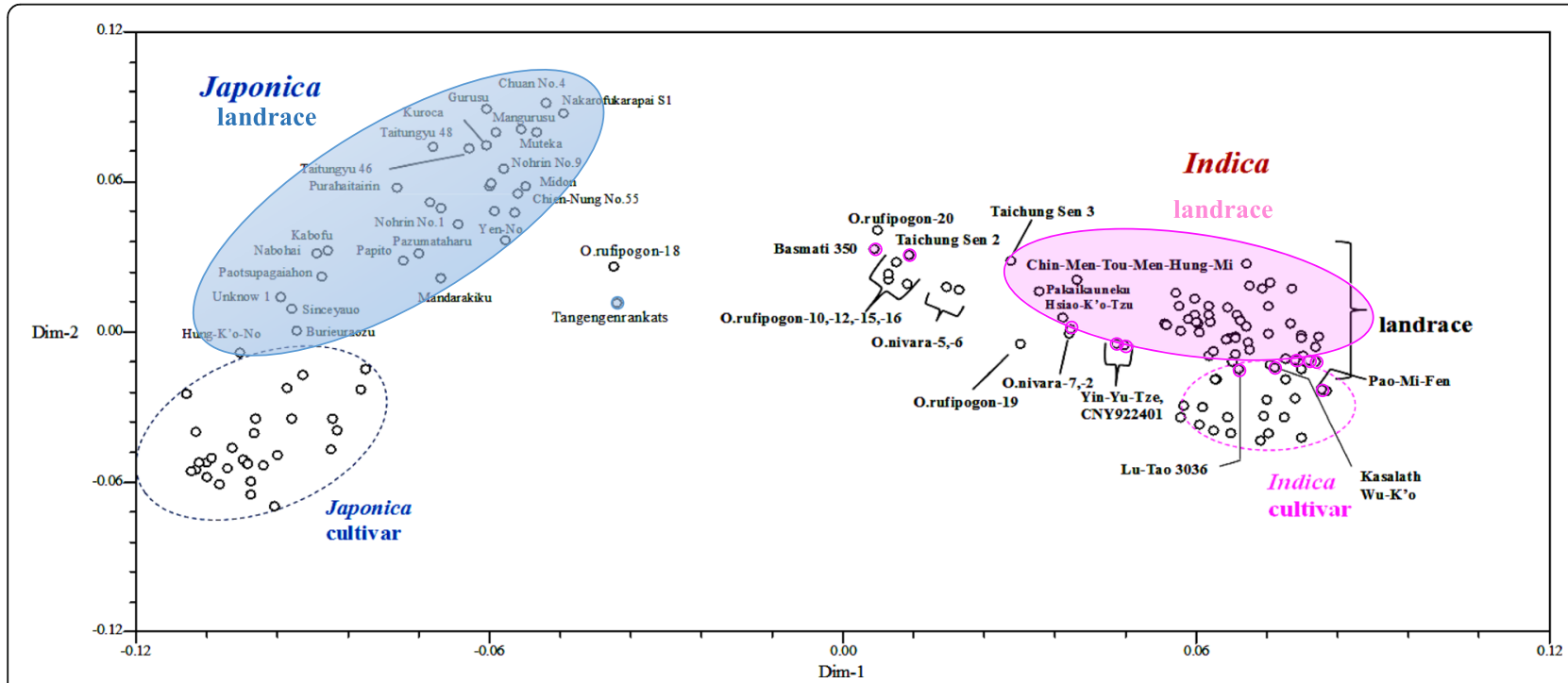

Fig. 1 Principle coordinate analysis (PCOA) of 148 rice accessions. After 2-dimension analysis of PCoA, the first and second dimension explained 18.1\% and $7.7 \%$, respectively. Each accession is indicated by a circle. Japonica and indica rices are distinctly separated, with japonica and indica landraces indicated by blue and magenta solid ellipses, and japonica and indica cultivars indicated by blue and magenta dashed ellipses, respectively 


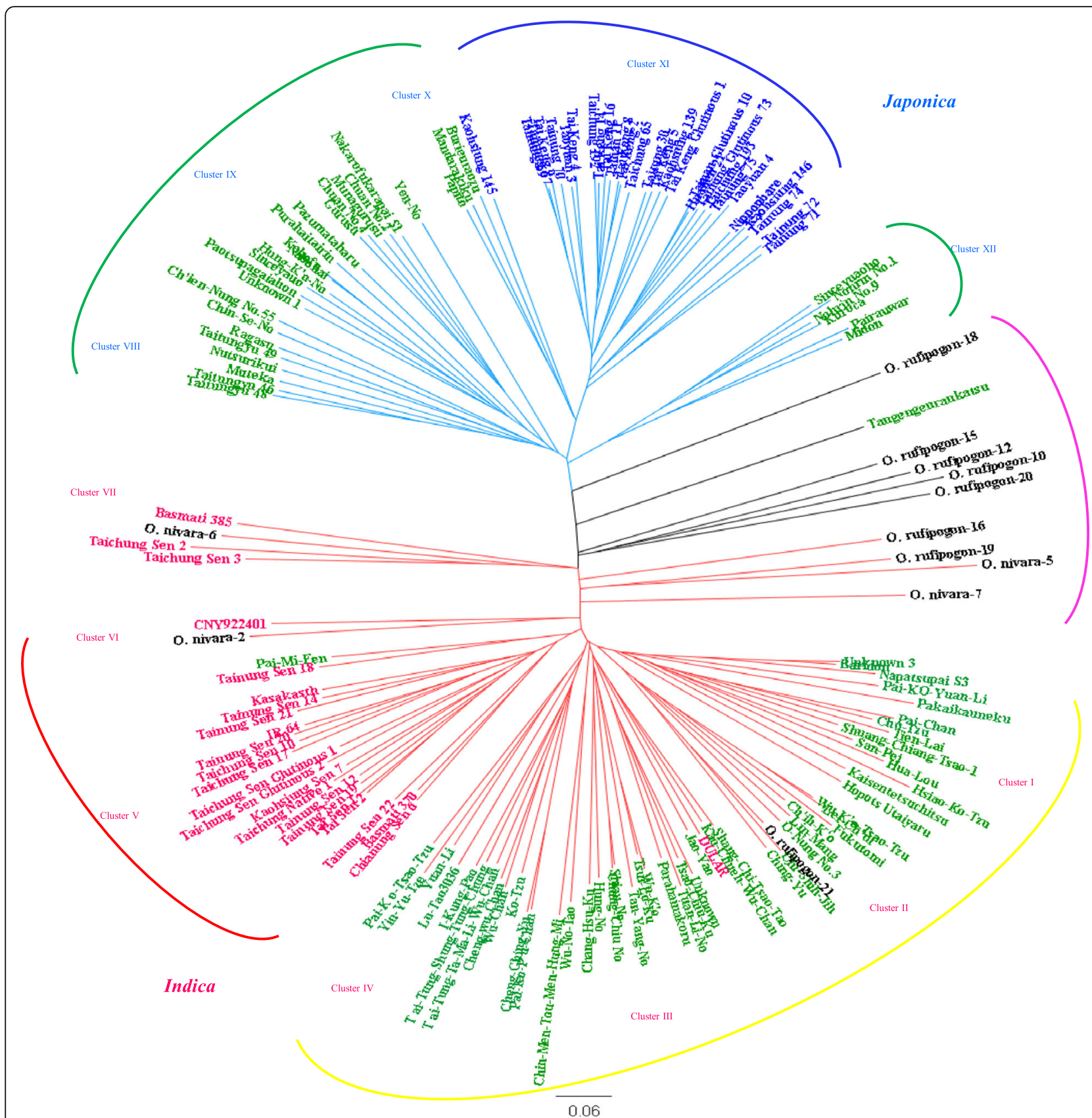

Fig. 2 Unrooted neighbor-joining tree of 148 rice accessions. Genetic distance was calculated according to Nei (1983) with the genotypes of 75 markers and cluster analysis by the neighbor-joining method. Japonica cultivars, indica cultivars, landrace and wild rices are indicated by blue, red, green and black, respectively. Cluster I-VII belong to indica sub-groups and Cluster VIII-XII belong to japonica sub-groups. Bar represents genetic distance

The Genetic Diversity and Differentiation in the Collected Rice Germplasm

The diversity panel of 148 accessions could be separated into two subpopulations according to STRUCTURE analysis, clearly corresponding to indica and japonica groups (Additional file 2: Figure S3a). Further division into five subpopulations, indica cultivars, indica landraces, japonica cultivars, japonica landraces and wild rice, were supported by $K=5$ (Table 1 , Additional file 2: Figure S3b). Most accessions were classified into the expected groups according to the records of the National Plant Genetic Resource Center (NPGRC) Taiwan that were classified by plant and seed morphology, however some were incongruent due to admixed genetic background. For example, landraces, Sinceyauo from Japan, Hung-K'o-No from China and Burieuraozu, Nabohai, 
Paotsupagaiahon, and Tangengenrankatsu from Taiwan, were grouped with japonica cultivars (Pop III); an aus cultivar (Dular) and O. rufipogon-21 were assigned to the indica landrace group (Popn IV); and three indica cultivars, Basmati 385, Taichung Sen 2 and Taichung Sen 3, were placed in the wild rice group (PopV) (Table 1). These accessions might still share identical by descent segments since derivation from common ancestors. One possible factor contributing to such incongruous findings that cannot be neglected is introgression owing to gene flow among wild species, landraces, and cultivars (Ishikawa et al. 2006; Wang et al. 2018). For example, a mega variety TC65 inherited photoperiodinsensitive alleles of Heading date 1 (Hd1) and Early heading date 1 (Ehd1) from two landraces, Muteke and Nakabo, by spontaneous introgression of natural gene flow during modern breeding (Wei et al. 2016). Indeed, landraces have been commonly used in breeding programs especially in the early purification breeding stage e.g. two old Taiwanese indica cultivars, Taichung Sen 2 and Taichung Sen 3, derived from landraces based on breeding records. Thus, admixed accessions are not necessarily rare outcomes of natural introgression, but derive from intentional cross hybridization in at least some cases.

Morphology and genetic background are quite different between indica and japonica rice through independent origins, long-term adaptation to diverse environments and selection for various human preferences. The extent of genetic differentiation between these two subspecies was revealed by $F_{\mathrm{ST}}$ analysis (Ikehashi 2009; Zhang et al. 2007). High genetic differentiation $\left(F_{\mathrm{ST}}=0.3084\right)$ was observed between indica and japonica groups in our rice diversity panel (Table 3), in agreement with several studies (Thomson et al. 2007; Lin et al. 2012). The level of differentiation between indica and japonica landraces $\left(F_{\mathrm{ST}}=0.3040\right)$ was lower than that between indica and japonica cultivars $\left(F_{\mathrm{ST}}=\right.$ $0.4200)$. Landraces were selected by farmers for adaptation to local environments and various preferences; while modern cultivars result from intense directional selection for specific traits. Less differentiation in landraces than in cultivars was associated with different selection intensity.

The gene diversity of indica accessions was higher than that of japonica accessions since the bottleneck effect was more severe in japonica rice during early domestication (Kovach and McCouch 2008; Wang et al. 2018; Zhu et al. 2007). In the present study, genetic diversity was much lower in japonica than indica populations as well (Fig. 1, Table 3), the same tendency as in previous studies using Taiwan breeding germplasm and a collection from Borneo Island (Lin et al. 2012; Thomson et al. 2009). Nevertheless, in our collection the level of diversity of japonica landraces was higher than that of indica landraces (Table 3) because the former included both upland and lowland accessions.

\section{Unveiling Taiwanese Rice Germplasm}

Today, indigenous peoples still cultivate their own landraces with unique traits, such as large grain and aroma, on upland fields in Taiwan. The cultivation of rice, accompanied by foxtail millet, can be dated back to 5000 years ago by unearthed grain remains from some archaeological sites in Tainan Science Park, southern Taiwan (Tsang 2012). Approximately $98 \%$ and $83 \%$ of the excavated carbonized rice grains from the Tapenkeng Culture period (4800-4200 B.P.) and Niuchoutzu Culture period (3800-3300 B.P), respectively, were classified as japonica rice according to grain morphology (Tsang 2012; Wang 2007).

In the present study, 17 landraces labeled with "\#) in the Additional file 1: Table S1, were grouped in Clusters VIII, IX, X and XII which belong to the japonica clade (Fig. 2). These indigenous landraces were genetically distinct from modern temperate japonica cultivars, Cluster XI (Fig. 2), and presumed to belong to tropical japonica rice (javanica). The upland landrace, Tangengenrankatsu, has admixed genetic background and is genetically close to O. rufipogon-18. Only few indigenous landraces were clustered in indica clades, albeit some were classified as japonica rice by morphology according to NPGR $\mathrm{C}$ records, such as Pakaikauneku, Kaisentetsuchitsu, Napatsupa S3, and Baridon (Additional file 1: Table S1, Fig. 2). Tropical japonica, diverged from temperate japonica, is thought to have originated in the upper ThaiMalay Peninsula and might have moved from the Malay Archipelago northward through Indonesia, the Philippines, Taiwan, Ryukyus, and Japan (Chang 1976; Gutaker et al. 2020). Thus, Taiwan was on the dispersal route of tropical japonica and $2 / 3$ of carbonized rice grains unearthed from remains of Niaosung Culture (1400-1000 B.P.) had grain length larger than $4 \mathrm{~mm}$ which resembled tropical japonica (Wang 2007). In accordance with archaeobotanical evidence, phylogenetic analysis of SSR genotypes classified indigenous upland landraces as tropical japonica (Fig. 2).

In the indica clusters, only 6 accessions were recorded with indigenous language pronunciations, including 5 (Baridon, Napatsupai S3, Pakaikameku, Kaisentetsuchitsu, Hopot Utatyaru) in cluster I and Parahainakoru in cluster III (Fig. 2). These 6 indica landraces might have been preserved and cultivated by indigenous people for thousands of years, however archaeobotanical evidence is lacking. We cannot rule out that these indica landraces were adopted by indigenous people only hundreds of years ago, after Chinese introduced much indica rice. The landraces in cluster II - IV came from 
Taiwan and China and showed no significant isolationby-distance (Fig. 2). However, the indica landraces were divided into two large clades, Cluster I \& II and Cluster III \& IV, which might reflect two origins, Guangdong and Fujian. The genetic diversity of indica landraces in Taiwan is relatively high (Tables 3,4 ) which might result from intrinsic high variation in indica rice and multiple origins as well. Taiwanese indica cultivars, closer to IR64 than Dular, an Aus cultivar in India (Fig. 2), might be resulted from modern breeding that 14 of 17 Taiwanese indica cultivars can be traced back to IRRI accessions or DGWG as their breeding parents ( $\mathrm{Lu}$ and $\mathrm{Lu}$ 2010).

Landraces, intermediate between wild relatives and cultivars, are important genetic reservoir for crop improvement to cope with climate changes and increase sustainability. In Taiwan, 16 officially acknowledged indigenous peoples have their own cultures and diet preferences, including diversified crop germplasm. Taiwanese rice landraces compromised of tropical japonica and indica rice revealed diverse genetic variation in plant architecture and seeds (Hsieh et al. 2011) and herein showed much SSR diversity (Tables 3, 4). This high genetic variation indicates that Taiwanese landraces are a reservoir of genetic diversity and beneficial genes/alleles for rice breeding and improvement. Taiwanese landraces have had great impact on modern rice breeding not only in Taiwan but also elsewhere in the world. According to the database of rice breeding pedigrees (Taiwan Rice Information System, TRIS), Taiwanese landraces were commonly used to introgress useful genes for rice improvement, especially in the early breeding programs a half-century ago. The most prominent varieties, japonica TC65 with photoperiod insensitivity and indica variety Taichung Native 1 (TCN1) with semi-dwarf stature, have had great impact on rice breeding and research. Because photoperiod insensitivity was a highly desired trait, TC65 had been extensively applied in modern rice breeding programs, leading to all current Taiwanese temperate japonica cultivars inheriting the ehd1 and $h d 1$ alleles. Taiwanese temperate japonica cultivars can be cultivated in two crop seasons under tropical and subtropical environments, making Taiwan the southernmost region of temperate japonica cultivation. The indica variety TCN1 inherited null function of $s d 1$ with a 383-bp deletion from the landrace DGWG (Sasaki et al. 2002), and, this DGWG allele has been widely applied to improve grain yield of both indica and japonica varieties in the past 50 years (Asano et al. 2011; Zhao et al. 2010). Yet, there are still numerous useful genes/ alleles existing in the genetic reservoir of Taiwanese landraces, for example conferring large grain size, aroma, and biotic and abiotic resistance. Untapped beneficial genes from landraces can help to breed new varieties for resilient and sustainable agriculture.
Modern cultivars are a result of intensive directional selection for specific traits which are frequently determined by government policy and demands of markets. In Taiwan, the major dining staple was indica rice before War World II but changed to temperate japonica rice because of government policy during Japanese occupation. Now, japonica rice is for dining; while indica rice is used for various food processing needs, such rice noodles, pudding, and cakes. Thus, japonica and indica improvement have different breeding goals. For indica rice, high yield with resistances to biotic and abiotic stresses are breeding goals; thus, diverse germplasm from landraces or introduced from other countries are commonly utilized as donor parents (Lin et al. 2012). Therefore, there was no obvious difference in genetic diversity and differentiation between early and late indica cultivars $\left(F_{\mathrm{ST}}=0.0045\right.$, Table 4$)$. On the other hand, the breeding goal of japonica rice was changed from high yield to premium grain quality that the germplasm used for improving different traits seemed to be associated with high differentiation between early and late japonica cultivars, $F_{\mathrm{ST}}=0.3751$ (Table 4). In order to improve grain quality, a few Japanese elite temperate japonica cultivars were introduced and used extensively in recurrent breeding crosses (Lin et al. 2012). This led to modern Taiwanese japonica cultivars being grouped at the same clade, Cluster XI with the Japanese elite cultivar, Nipponbare (Fig. 2), as japonica varieties from Taiwan and Japan did not differ significantly in the pattern of genetic diversity (Lin et al. 2012). The genetic distances between any two Taiwanese japonica cultivars were in the range of $0.43-0.58$ (Fig. 2); consequently, the gene pool of japonica cultivars is relative narrow as compared to either japonica landraces or modern indica cultivars (Tables 3, 4), resulting in genetic vulnerability in rice cultivation and management.

To overcome severe genetic vulnerability of temperate japonica cultivars, wild relatives and indica rice were introduced to breeding programs. For example, japonica Tainung 67 was the descent of a cross of japonica Tainung 61 and $O$. rufipogon, and japonica Taichung 192 was an indicaljaponica-crossed variety (Lu and Lu 2010). Recently, numerous advanced breeding lines introduced from IRRI and wild relatives have been used in breeding programs to improve biotic and abiotic stresses for sustainable agriculture, e. g. IRBB66 pyramided with 5 bacterial blight resistant genes (Yap et al. 2016). Thus, current rice breeding goals in Taiwan emphasize grain quality first, followed by other traits such as resistances and multi-dimensional utilizations (forage and landscape). To achieve various goals, germplasm for breeding are not limited to the domestics but also exotics.

\section{Conclusions}

A diversity panel of 148 rice accessions, including 47 cultivars and 59 landraces from Taiwan and 42 
accessions from other countries, could be grouped into five major subpopulations: wild rices, japonica landraces, indica landraces, indica cultivars, and japonica cultivars. The genetic diversities, without exception, were wild rices $>$ landraces $>$ cultivars, and indica rice > japonica rice. The majority of Taiwanese japonica landraces preserved by indigenous peoples were classified as tropical japonica by morphology and phylogenetic analysis, consistent with archaeobotanical evidence. Thus, japonica landraces had greater genetic variation than indica landraces. The Taiwanese indica landraces could be separated into two clusters on phylogenetic trees, reflecting two sets of introductions from China. The genetic variation and divergence of modern cultivars are largely influenced by government policies and market demands, exemplified by premium grain quality for japonica rice, and yield and resistances for indica rice. Large genetic diversification was unveiled in Taiwanese landraces, as well as intermediate and admixed genetic background, providing a precious and valuable genetic reservoir for rice breeding in the future.

\section{Materials and Methods \\ Plant Materials}

A diversity panel of 148 rice accessions, including $136 O$. sativa, 4 O. nivara, and 8 O. rufipogon, were analyzed in this study. The germplasm originated from Taiwan, Japan, and China or was introduced from the International Rice Research Institute (IRRI), and was obtained from the National Plant Genetic Resource Center (NPGRC), Taiwan. These germplasms were propagated and used for rice improvement by rice breeders, Dr. Chih-Shen Sheu in Taichung District Agricultural Research and Extension Station and Dr. Yong-pei Wu in Chiayi Agricultural Experiment Branch, Taiwan Agricultural Research Institute. Each accession denoted indica or japonica and cultivars or landraces was in accordance with the record in NPGRC based on the classification according to morphology and collection sites. For cultivars, there were 29 japonica varieties including 28 Taiwanese and 1 Japanese cultivar and 24 indica varieties including 19 Taiwanese, 1 Pakistani and 3 Indian cultivars, and IR64. For landraces, there were 59, 18, and 6 accessions from Taiwan, China and Japan, respectively. For the 59 Taiwanese landraces, 23 accessions were recorded with pronunciations of indigenous languages, 36 with Chinese characters, and 3 labeled as Unknown, Unknown 1 and Unknown 3, respectively. The 12 wild rices were collected from China, Bangladesh and Laos (Additional file 1: Table S1).

\section{DNA Extraction and Genotyping Assay}

Genomic DNA was extracted from leaf tissues of rice seedlings at the three-leaf stage as described previously (Lin et al. 2012). A total of 75 markers including 49 published SSRs (McCouch et al. 2002), 6 STSs (Wu et al.
2010), and 20 newly-designed SSRs and indels (Additional file 1: Table 2) distributed across the rice genome were applied for genotyping assay.

Among the 75 markers, 56 were analyzed with a QIAxcl System -GT12 ${ }^{\mathrm{mm}}$ Genetic Analyzer (Qiagen, USA). The PCR reaction was in a total volume of $15 \mu \mathrm{L}$ containing $30 \mathrm{ng}$ genomic DNA, $0.3 \mathrm{nmol} / \mu \mathrm{L}$ forward and reverse primer each, and $8 \mu \mathrm{L}$ Taq DNA Polymerase Master Mix (Ampliqon, Denmark). Amplification was performed on a thermocycler (Model T1, Biometra, Germany) with the following thermal profile: $94^{\circ} \mathrm{C}$ for 3 min for 1 cycle; $94^{\circ} \mathrm{C}$ for $40 \mathrm{~s}$, $55^{\circ} \mathrm{C}$ for $40 \mathrm{~s}, 72^{\circ} \mathrm{C}$ for $40 \mathrm{~s}$, for 35 cycles; $72^{\circ} \mathrm{C}$ for $3 \mathrm{~min}$ for 1 cycle. Amplicons were resolved by QIAxcel DNA High Resolution Kit (1200) with QX size marker 25-450 and QX alignment marker $15 \mathrm{bp} / 500 \mathrm{bp}$ (Qiagen, USA). The other 19 markers were assessed on an ABI 3730 DNA Analyzer (Applied BioSystems, USA). PCR reactions were set in a total volume of $20 \mu \mathrm{L}$ containing $20 \mathrm{ng}$ of genomic DNA, $10 \mathrm{pmol} / \mu \mathrm{L}$ of primer labeled with a fluorescent dye, $2 \mu \mathrm{L}$ of $10 \times$ PCR buffer, $2 \mu \mathrm{L}$ of $2.5 \mathrm{nmol} / \mu \mathrm{L}$ dNTPs, $1.5 \mu \mathrm{L}$ of $5 \mathrm{U} / \mu \mathrm{L}$ Amplitaq Gold ${ }^{\circ}$ DNA polymerase (Applied Biosystems, USA), and $2 \mu \mathrm{L}$ of $1 \mathrm{~mol} / \mathrm{L}$ betaine. Amplifications were performed with the following thermal profile: $94^{\circ} \mathrm{C}$ for $5 \mathrm{~min}$ for $1 \mathrm{cycle} ; 95^{\circ} \mathrm{C}$ for $30 \mathrm{~s}, 55^{\circ} \mathrm{C}$ for $55 \mathrm{~s}, 72^{\circ} \mathrm{C}$ for $35 \mathrm{~s}$, for 35 cycles; and $72^{\circ} \mathrm{C}$ for $1 \mathrm{~min}$ for 1 cycle. DNA fragment analysis of amplified products were carried out by using an ABI 3730 DNA Analyzer with ABI GeneS$\operatorname{can}^{\text {TM }}-600$ LIZ $^{\mathrm{ma}}$ Size Standard following the manufacturer's instructions (Applied BioSystems, USA).

\section{Data Analysis}

To evaluate genetic relatedness of these 148 accessions, genotypes of 75 markers were subjected to genetic diversity, population structure simulation, principle coordinate analysis (PCoA), and phylogenetic analysis. Five genetic diversity parameters including mean allele number per locus, major allele frequency per locus, Nei's gene diversity, mean polymorphic information content (PIC), and fixation index $\left(F_{\mathrm{ST}}\right)$ were assessed by using PowerMarker V3.25 (Liu and Muse 2005).

Population structures of 148 accessions were analyzed by STRUCTURE V 2.3.3, a Bayesian model-based approach (Pritchard et al. 2000). Simulation was performed under the admixture model with 100,000 burn-in iterations of Markov Chain Monte Carlo (MCMC) for $K$ values set from 1 to 11 , and $\Delta K$ (an ad hoc quantity) was used to determine subpopulation number (Evanno et al. 2005).

The genetic distance of similarity matrix was calculated using modified Rogers' distance (Goodman and Stuber 1983). The genetic distances were consequently subjected to two-dimension principle coordinate analysis with Decnter and Eigene modules (Rohlf 1987) and used in construction of an unrooted phylogenetic tree by neighbor-joining in PowerMarker V3.25 (Liu and Muse 2005) and visualized using TreeView. 


\section{Additional Files}

Additional file 1: Table S1. Name, type, subspecies/species and origin of 148 rice accessions used in this study. Table S2. Chromosomal position, locus name and PIC value of 75 SSR marker used for this study.

Additional file 2: Figure S1. The frequency distribution of allele number and polymorphic information content (PIC) with 75 molecular markers. (A) Allele number per locus ranges from 3 to 37 with an average of 12.7. (B) PIC ranges from 0.18 to 0.95 with an average of 0.72 . Figure S2. (a) Structure simulation analysis to determine best $K$. (A) $L n P(D)$, the log likelihood for each K, was calculated by 100,000 permutations and mean $L n P(D)$ value was taken from 10 replications. $\Delta K$, an ad hoc quantity, is transferred by mean $\operatorname{LnP}(D)$ value and $\Delta K$ of 148 accessions. (B) $\operatorname{LnP}(\mathrm{D})$ value and $\Delta K$ of 86 indica accessions. $(C) \operatorname{LnP}(\mathrm{D})$ value and $\Delta K$ of 86 japonica accessions. Figure S3. Population structure analysis of 148 accessions. Each individual is indicated by a vertical bar. (A) For $K=2$, pop2-1 and pop2-2, indicated by red and green, are composed of japonica and indica rice, respectively. (B) For $K=5$, pop5-1, pop5-2, pop5-3, pop5-4 and pop5-5, indicated by red, green, blue, yellow and magenta, are composed of indica cultivar, japonica landrace, japonica cultivar, indica landraces and wild rices, respectively. The numbers of accessions in each subpopulation are indicated in brackets (). Figure S4. Three-dimensional plot from principle coordinate analysis of 148 rice accessions. Japonica and indica are separated on opposite sides. Japonica and indica cultivars are marked with circles.

\section{Abbreviations}

IRRI: International Rice Research Institute; SNP: Single nucleotide polymorphism; DGWG: Dee-Geo-Woo-Gen; PCoA: Principal coordinate analysis; SSR: Simple sequence repeat; STS: Sequence-tagged site; indel: Insertion/deletion; PIC: Polymorphic information content; Pop: Population; NPGRC: National Plant Genetic Resource Center; TC65: Taichung 65; TCN1: Taichung Native 1

\section{Acknowledgements}

The authors are thankful to Dr. Chih-Shen Sheu for providing some germplasm. We also appreciate the services of Professor Hawkeye, LLC for critical review and English editing.

\section{Authors' Contribution}

$\mathrm{AH}$ and $\mathrm{YL}$ designed and supervised the research; $\mathrm{YW}$ prepared genetic materials; WH and SC conducted genotype assay and analyzed data; $\mathrm{AH}, \mathrm{WH}$, and $Y L$ wrote the manuscript. $A H, H C$, and $Y L$ edited and revised the manuscript. All authors read and approved the final manuscript.

\section{Funding}

This work was supported by the National Science Council (NSC 98-2324-B002-001) of Taiwan.

\section{Availability of Data and Materials}

Dataset and figures supporting the results are included as additional files.

\section{Ethics Approval and Consent to Participate}

Not applicable.

\section{Consent for Publication}

All authors have provided consent for publication.

\section{Competing Interests}

The authors declare that they have no competing interests.

\section{Author details}

${ }^{1}$ Department of Life Science, Fu-Jen Catholic University, New Taipei City 242062, Taiwan. ${ }^{2}$ Department of Agronomy, National Taiwan University, Taipei 10617, Taiwan. ${ }^{3}$ Department of Agronomy, Chiayi Agricultural Experiment Branch, Taiwan Agricultural Research Institute, Chiayi 600015, Taiwan.
Received: 17 July 2020 Accepted: 6 December 2020

Published online: 14 December 2020

\section{References}

Asano K, Yamasaki M, Takuno S, Miura K, Katagiri S, Ito T, Doi K, Wu J, Ebana K, Matsumoto T, Innan H, Kitano H, Ashikari M, Matsuoka M (2011) Artificial selection for a green revolution gene during japonica rice domestication. Proc Natl Acad Sci U.S.A 108(27):11034-11039. https://doi.org/10.1073/pnas. 1019490108

Caicedo AL, Williamson SH, Hernandez RD, Boyko A, Fledel-Alon A, York TL, Polato NR, Olsen KM, Nielsen R, McCouch SR, Bustamante CD, Purugganan MD (2007) Genome-wide patterns of nucleotide polymorphism in domesticated rice. PLoS Genet 3(9):1745-1756. https://doi.org/10.1371/ journal.pgen.0030163

Chakhonkaen S, Pitnjam K, Saisuk W, Ukoskit K, Muangprom A (2012) Genetic structure of Thai rice and rice accessions obtained from the international rice research institute. Rice 5(1):19. https://doi.org/10.1186/1939-8433-5-19

Chang $T$ (1976) The origin, evolution, cultivation, dissemnination, and diversificaiton of Asian and Africa rice. Euphytica 25:425-441

Chin HS, Wu YP, Hour AL, Hong CY, Lin YR (2016) Genetic and evolutionary analysis of purple leaf sheath in rice. Rice 9(1):8. https://doi.org/10.1186/ s12284-016-0080-y

Choi JY, Platts AE, Fuller DQ, Hsing YL, Wing RA, Purugganan MD (2017) The rice paradox: multiple origins but single domestication in Asian rice. Mol Biol Evol 34(4):969-979. https://doi.org/10.1093/molbev/msx049

Choi JY, Purugganan MD (2018) Multiple origin but single domestication led to Oryza sativa. G3 (Bethesda) 8(3):797-803. https://doi.org/10.1534/g3.117. 300334

Chou SL (1948) China is the place of origin of rice. J Rice Soc China 7:53-54 (in Chinese)

Courtois B, Audebert A, Dardou A, Roques S, Ghneim-Herrera T, Droc G, Frouin J, Rouan L, Gozé E, Kilian A, Ahmadi N, Dingkuhn M (2013) Genome-wide association mapping of root traits in a japonica rice panel. PLoS One 8(11): e78037. https://doi.org/10.1371/journal.pone.0078037

Doi K, Izawa T, Fuse T, Yamanouchi U, Kubo T, Shimatani Z, Yano M, Yoshimura A (2004) Ehd1, a B-type response regulator in rice, confers short-day promotion of flowering and controls FT-like gene expression independently of $\mathrm{Hd} 1$. Genes Dev 18(8):926-936. https://doi.org/10.1101/gad.1189604

Dwivedi SL, Ceccarelli S, Blair MW, Upadhyaya HD, Are AK, Ortiz R (2016) Landrace germplasm for improving yield and abiotic stress adaptation. Trends Plant Sci 21(1):31-42. https://doi.org/10.1016/j.tplants.2015.10.012

Evanno G, Regnaut S, Goudet J (2005) Detecting the number of clusters of individuals using the software STRUCTURE: a simulation study. Mol Ecol 14(8): 2611-2620. https://doi.org/10.1111/j.1365-294X.2005.02553.x

Evenson RE, Gollin D (2003) Assessing the impact of the green revolution, 1960 to 2000. Science 300(5620):758-762. https://doi.org/10.1126/science.1078710

Fuentes RR, Chebotarov D, Duitama J, Smith S, De la Hoz JF, Mohiyuddin M, Wing RA, McNally KL, Tatarinova T, Grigoriev A, Mauleon R, Alexandrov N (2019) Structural variants in 3000 rice genomes. Genome Res 29(5):870-880. https://doi.org/10.1101/gr.241240.118

Garris AJ, Tai TH, Coburn J, Kresovich S, McCouch S (2005) Genetic structure and diversity in Oryza sativa L. Genetics 169(3):1631-1638. https://doi.org/10.1534/ genetics.104.035642

Goodman MM, Stuber CW (1983) Races of maize: VI. Isozyme variation among races of maize in Bolivia Maydica 28:169-187

Gutaker RM, Groen SC, Bellis ES, Choi JY, Pires IS, Bocinsky RK, Slayton ER, Wilkins O, Castillo CC, Negrao S, Oliveira MM, Fuller DQ, Guedes JAD, Lasky JR, Purugganan MD (2020) Genomic history and ecology of the geographic spread of rice. Nat Plants 6(5):492-502. https://doi.org/10.1038/s41477-0200659-6

Hsieh JS, Hsing YI, Hsu TF, Li JK, Li KT, Tsang CH (2011) Studies on ancient rice-where botanists, agronomists, archeologists, linguists, and ethnologists meet. Rice 4(3-4):178-183. https://doi.org/10.1007/s12284-011-9075-x

Hu CW (1993) Historical review of semidwarf Rices and breeding of a new plant type for sustainable agriculture. Res Bull Taichung Dist Agric Improv Stn 38: $45-63$

Huang X, Wei X, Sang T, Zhao Q, Feng Q, Zhao Y, Li C, Zhu C, Lu T, Zhang Z, Li M, Fan D, Guo Y, Wang A, Wang L, Deng L, Li W, Lu Y, Weng Q, Liu K, Huang T, Zhou T, Jing Y, Li W, Lin Z, Buckler ES, Qian Q, Zhang QF, Li J, Han B (2010) Genome-wide association studies of 14 agronomic traits in rice landraces. Nat Genet 42(11):961-967. https://doi.org/10.1038/ng.695 
Ikehashi $\mathrm{H}$ (2009) Why are there indica type and japonica type in rice? - history of the studies and a view for origin of two types. Rice Sci 16:1-13. https:// doi.org/10.1016/S1672-6308(08)60050-5

Ishikawa R, Yamanaka S, Fukuta Y, Chitrakon S, Bounphanousay C, Kanyavong K, Tang LH, Nakamura I, Sato T, Sato YI (2006) Genetic erosion from modern varieties into traditional upland rice cultivars (Oryza sativa L.) in northern Thailand. Genet Resour Crop Evol 53:245-252. https://doi.org/10.1007/ s10722-004-6132-y

Iso E (1964) Talks on Horai rice. Amayomikai, Yamakuchi, p 89 in Japanese

Jin L, Lu Y, Xiao P, Sun M, Corke H, Bao J (2010) Genetic diversity and population structure of a diverse set of rice germplasm for association mapping. Theor Appl Genet 121(3):475-487. https://doi.org/10.1007/s00122-010-1324-7

Khush GS (1997) Origin, dispersal, cultivation and variation of rice. Plant Mol Biol 35(1-2):25-34

Kovach MJ, McCouch SR (2008) Leveraging natural diversity: back through the bottleneck. Curr Opin Plant Biol 11(2):193-200. https://doi.org/10.1016/j.pbi. 2007.12 .006

Li JY, Wang J, Zeigler RS (2014) The 3,000 rice genomes project: new opportunities and challenges for future rice research. Gigascience 3:8. https:// doi.org/10.1186/2047-217X-3-8

Lin HY, Wu YP, Hour AL, Ho SW, Wei FJ, Hsing YC, Lin YR (2012) Genetic diversity of rice germplasm used in Taiwan breeding programs. Bot Stud 53:363-376

Lin MS (1991) Field uniformity of the japonica rice region of Taiwan as estimated by relative genetic contribution. Theor Appl Genet 83:115-118

Liu K, Muse SV (2005) PowerMarker: an integrated analysis environment for genetic marker analysis. Bioinformatics 21(9):2128-2129. https://doi.org/10. 1093/bioinformatics/bti282

Londo JP, Chiang YC, Hung KH, Chiang TY, Schaal BA (2006) Phylogeography of Asian wild rice, Oryza rufipogon, reveals multiple independent domestications of cultivated rice, Oryza sativa. Proc Natl Acad Sci U.S.A 103(25):9578-9583. https://doi.org/10.1073/pnas.0603152103

Lu CT, Lu HY (2010) Establishment and application of Taiwan rice information system. J Taiwan Agric Res 59:61-69 (Chinese with English abstract)

McCouch SR, Teytelman L, Xu YB, Lobos KB, Clare K, Walton M, Fu BY, Maghirang R, Li ZK, Xing YZ, Zhang QF, Kono I, Yano M, Fjellstrom R, DeClerck G, Schneider D, Cartinhour S, Ware D, Stein L (2002) Development and mapping of 2240 new SSR markers for rice (Oryza sativa L.). DNA Res 9(6): 199-207. https://doi.org/10.1093/dnares/9.6.199

McNally KL, Childs KL, Bohnert R, Davidson RM, Zhao K, Ulat VJ, Zeller G, Clark RM, Hoen DR, Bureau TE, Stokowski R, Ballinger DG, Frazer KA, Cox DR, Padhukasahasram B, Bustamante CD, Weigel D, Mackill DJ, Bruskiewich RM, Ratsch G, Buell CR, Leung H, Leach JE (2009) Genomewide SNP variation reveals relationships among landraces and modern varieties of rice. Proc Natl Acad Sci U.S.A 106(30):12273-12278. https://doi.org/10.1073/pnas.0900992106

Nachimuthu W, Muthurajan R, Duraialaguraja S, Sivakami R, Pandian BA, Ponniah G, Gunasekaran K, Swaminathan M, Suji KK, Sabariappan R (2015) Analysis of population structure and genetic diversity in rice germplasm using SSR markers: an initiative towards association mapping of agronomic traits in Oryza sativa. Rice 8(1):30. https://doi.org/10.1186/s12284-015-0062-5

Pritchard JK, Stephens M, Donnelly P (2000) Inference of population structure using multilocus genotype data. Genetics 155:945-959

Pusadee T, Jamjod S, Chiang YC, Rerkasem B, Schaal BA (2009) Genetic structure and isolation by distance in a landrace of Thai rice. Proc Natl Acad Sci U.S.A 106(33):13880-13885. https://doi.org/10.1073/pnas.0906720106

Rohlf F (1987) NTSYS-pc: microcomputer programs for numerical taxonomy and multivariate analysis. Am Stat 41:330. https://doi.org/10.2307/2684761

Sang T, Ge S (2013) Understanding rice domestication and implications for cultivar improvement. Curr Opin Plant Biol 16(2):139-146. https://doi.org/10. 1016/j.pbi.2013.03.003

Sasaki A, Ashikari M, Ueguchi-Tanaka M, Itoh H, Nishimura A, Swapan D, Ishiyama K, Saito T, Kobayashi M, Khush GS, Kitano H, Matsuoka M (2002) Green revolution: a mutant gibberellin-synthesis gene in rice. Nature 416(6882):701702. https://doi.org/10.1038/416701a

Thomson MJ, Polato NR, Prasetiyono J, Trijatmiko KR, Silitonga TS, McCouch SR (2009) Genetic diversity of isolated populations of indonesian landraces of rice (Oryza sativa L.) collected in East Kalimantan on the island of Borneo. Rice 2(1):80-92. https://doi.org/10.1007/s12284-009-9023-1

Thomson MJ, Septiningsih EM, Suwardjo F, Santoso TJ, Silitonga TS, McCouch SR (2007) Genetic diversity analysis of traditional and improved Indonesian rice (Oryza sativa L.) germplasm using microsatellite markers. Theor Appl Genet 114(3):559-568. https://doi.org/10.1007/s00122-006-0457-1
Tsang CH (2012) Issues relating to the ancient rice and millet grains unearthed from the archaeological sites in Tainan Science Park. J Chin Dietary Culture 8: 1-14 (Chinese with English abtract)

Wang W, Mauleon R, Hu Z, Chebotarov D, Tai S, Wu Z, Li M, Zheng T, Fuentes RR, Zhang F, Mansueto L, Copetti D, Sanciangco M, Palis KC, Xu J, Sun C, Fu B, Zhang H, Gao Y, Zhao X, Shen F, Cui X, Yu H, Li Z, Chen M, Detras J, Zhou Y, Zhang X, Zhao Y, Kudrna D, Wang C, Li R, Jia B, Lu J, He X, Dong Z, Xu J, Li Y, Wang M, Shi J, Li J, Zhang D, Lee S, Hu W, Poliakov A, Dubchak I, Ulat VJ, Borja FN, Mendoza JR, Ali J, Li J, Gao Q, Niu Y, Yue Z, Naredo MEB, Talag J, Wang X, Li J, Fang X, Yin Y, Glaszmann JC, Zhang J, Li J, Hamilton RS, Wing RA, Ruan J, Zhang G, Wei C, Alexandrov N, McNally KL, Li Z, Leung H (2018) Genomic variation in 3,010 diverse accessions of Asian cultivated rice. Nature 557(7703):43-49. https://doi.org/10.1038/s41586-018-0063-9

Wang YH (2007) The preliminary notes on the ancient rice grains excavated in Taiwan. Master thesis. Department of Agronomy, National Taiwan University, Taipei, Taiwan. (Chinese with English abstract)

Wei FJ, Tsai YC, Wu HP, Huang LT, Chen YC, Chen YF, Wu CC, Tseng YT, Hsing YC (2016) Both $\mathrm{Hd} 1$ and Ehd1 are important for artificial selection of flowering time in cultivated rice. Plant Sci 242:187-194. https//doi.org/10.1016/j.plantsci.2015.09.005

Wu YP, Ko PY, Lee WC, Wei FJ, Kuo SC, Ho SW, Hour AL, Hsing YI, Lin YR (2010) Comparative analyses of linkage maps and segregation distortion of two $F_{2}$ populations derived from japonica crossed with indica rice. Hereditas 147(5): 225-236. https://doi.org/10.1111/j.1601-5223.2010.02120.x

Yano M, Katayose Y, Ashikari M, Yamanouchi U, Monna L, Fuse T, Baba T, Yamamoto K, Umehara Y, Nagamura Y, Sasaki T (2000) Hd1, a major photoperiod sensitivity quantitative trait locus in rice, is closely related to the arabidopsis flowering time gene CONSTANS. Plant Cell 12(12):2473-2483. https://doi.org/10.1105/tpc.12.12.2473

Yap R, Hsu YC, Wu YP, Lin YR, Kuo CW (2016) Multiplex PCR genotyping for five bacterial blight resistance genes applied to marker-assisted selection in rice (Oryza sativa). Plant Breed 135:309-317

Zhang D, Zhang H, Wang M, Sun J, Qi Y, Wang F, Wei X, Han L, Wang X, Li Z (2009) Genetic structure and differentiation of Oryza sativa L. in China revealed by microsatellites. Theor Appl Genet 119(6):1105-1117. https://doi. org/10.1007/s00122-009-1112-4

Zhang H, Sun J, Wang M, Liao D, Zeng Y, Shen S, Yu P, Mu P, Wang X, Li Z (2007) Genetic structure and phylogeography of rice landraces in Yunnan, China, revealed by SSR. Genome 50(1):72-83. https://doi.org/10.1139/g06-130

Zhao KY, Wright M, Kimball J, Eizenga G, McClung A, Kovach M, Tyagi W, Ali ML, Tung CW, Reynolds A, Bustamante CD, McCouch SR (2010) Genomic diversity and introgression in $O$. sativa reveal the impact of domestication and breeding on the rice genome. PLoS One 5(5):e10780

Zhao Q, Feng Q, Lu H, Li Y, Wang A, Tian Q, Zhan Q, Lu Y, Zhang L, Huang T, Wang Y, Fan D, Zhao Y, Wang Z, Zhou C, Chen J, Zhu C, Li W, Weng Q, Xu Q, Wang ZX, Wei X, Han B, Huang X (2018) Pan-genome analysis highlights the extent of genomic variation in cultivated and wild rice. Nat Genet 50(2): 278-284. https://doi.org/10.1038/s41588-018-0041-z

Zhu Q, Zheng X, Luo J, Gaut BS, Ge S (2007) Multilocus analysis of nucleotide variation of Oryza sativa and its wild relatives: severe bottleneck during domestication of rice. Mol Biol Evol 24(3):875-888. https://doi.org/10.1093/molbev/msm005

\section{Publisher's Note}

Springer Nature remains neutral with regard to jurisdictional claims in published maps and institutional affiliations. 